\title{
Prostatitis: revisión de una patología enigmática y su relación con la fertilidad masculina
}

\section{Prostatitis: Review of an Enigmatic Pathology and its Relation to Male Fertility}

\author{
Jenniffer Puerta Suárez ${ }^{1}$ Walter D. Cardona Maya ${ }^{1}$ \\ ${ }^{1}$ Grupo Reproducción, Departamento de Microbiología y \\ Parasitología, Facultad de Medicina, Universidad de Antioquia, \\ Medellín, Antioquia, Colombia \\ Urol Colomb 2018;27:233-242.

\begin{abstract}
Address for correspondence Walter D. Cardona Maya, PhD, Grupo Reproducción, Departamento de Microbiología y Parasitología, Facultad de Medicina, Universidad de Antioquia, Carrera 53 \# 61-30, Sede de Investigación Universitaria, SIU; Laboratorio 534, Medellín, Antioquia, Colombia (e-mail: wdario.cardona@udea.edu.co).
\end{abstract}

\section{Resumen \\ Palabras Clave \\ - prostatitis \\ - síndrome de dolor pélvico crónico \\ - infección \\ - prevalencia \\ - diagnóstico}

Introducción La prostatitis, término que involucra cuatro categorías de inflamación de la próstata, es considerada una patología enigmática por su complejidad en el diagnóstico e involucra el uso indiscriminado de antibióticos sin obtener evidencia de la infección. El objetivo de esta revisión es describir una patología de mal diagnóstico y de gran magnitud sobre la fertilidad masculina.

Materiales y Métodos Se realizó una revisión de la literatura científica a conveniencia en la base de datos PubMed, empleando los términos prostatitis, prevalencia, dolor pélvico crónico y diagnóstico.

Resultados La prevalencia de prostatitis varía entre $1,8 \%$ y $65 \%$ en los estudios evaluados, la prevalencia depende de la metodología empleada y del tipo de población evaluada.

Conclusión Se requiere determinar la prevalencia de prostatitis en la población en general, con el fin de mejorar las ayudas diagnósticas y el tratamiento para evitar la propagación de microorganismos.

Introduction Prostatitis, a term that involves four categories of inflammation of the prostate, is considered an enigmatic pathology because of its complexity in diagnosis and involves an indiscriminate use of antibiotics without obtaining evidence of infection. The objective of this review is to describe pathology with a poor diagnosis and great magnitude on the male fertility.

Materials and Methods A review of the scientific literature was performed at the PubMed database using the terms prostatitis, prevalence, chronic pelvic pain and diagnosis.

Results The prevalence of prostatitis varies between $1.8 \%$ and $65 \%$; the prevalence depends on the methodology used and the type of population evaluated in each study. Conclusion It is necessary to determine the prevalence of prostatitis in general population to improve diagnostic aid and treatment to prevent the spread of microorganisms. received

March 29, 2017

accepted

September 9, 2017

published online

May 25, 2018
DOI https://doi.org/

10.1055/s-0038-1656520.

ISSN 0120-789X.

eISSN 2027-0119.
Copyright ( 2018, Sociedad Colombiana License terms de Urología. Publicado por Thieme Revinter Publicações Ltda., Rio de Janeiro, Brazil. Todos los derechos reservados.

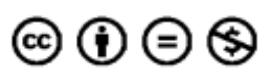




\section{Introducción}

La prostatitis, considerada una patología enigmática por su complejidad en el diagnóstico, involucra el uso indiscriminado de antibióticos sin tener evidencia de la infección. Siendo la eyaculación una cascada sincronizada de eventos que permiten que los espermatozoides interactúen con el oocito, ${ }^{1}$ todos los componentes del semen deben estar en óptimas condiciones para hacer más eficiente el proceso, sin embargo, el semen contiene células espermáticas y plasma seminal, en el que pueden alojarse una amplia diversidad de microorganismos e interferir durante la fecundación. Los microorganismos presentes en el semen provienen de diferentes localizaciones anatómicas e inclusive pueden ser microbiota del tracto genitourinario o microorganismos patógenos que desencadenan procesos infecciosos con secuelas sobre la fertilidad masculina como es el caso de la prostatitis bacteriana. El objetivo de esta revisión es describir una patología de mal diagnóstico y de gran magnitud sobre la fertilidad masculina, como es la prostatitis.

\section{Materiales y Métodos}

Se realizó una revisión a conveniencia de la literatura en la base de datos PubMed (National Center for Biotechnology Information - NCBI), empleando los siguientes términos para la búsqueda: prostatitis, prevalencia, dolor pélvico crónico y diagnóstico. De las 728 publicaciones encontradas, se seleccionaron para esta revisión las de mayor interés a criterio de los autores.

\section{Anatomía y Fisiología de la Próstata}

Antes de iniciar la descripción de la alteración prostática, es importante recordar que la próstata es una glándula dividida en tres zonas: la central, la de transición y la periférica. La zona central se encuentra sobre la base de la vejiga, ${ }^{1}$ por lo tanto, cuando se inflama, ejerce presión sobre ese órgano ocasionando las molestias miccionales asociadas a la prostatitis.

La glándula prostática tiene gran variedad de mecanismos de defensa contra la infección entre los que se encuentra la producción de sustancias antimicrobianas, el lavado mecánico de la uretra prostática por la micción y la eyaculación, sin embargo, el mal drenaje de las secreciones de los conductos distales o reflujo de orina en el tejido de la próstata puede conducir a la inflamación, cicatrización o formación de cálculos, por lo que la mayoría de las prostatitis bacterianas son precedidas por infecciones del tracto urinario. $^{2}$

\section{Funciones de Una Próstata Sana}

La próstata es una glándula masculina unida a la vejiga responsable de secretar fluido prostático rico en zinc, que representa entre el $25 \%$ y $30 \%$ del volumen del eyaculado. ${ }^{3} \mathrm{La}$ principal contribución de las glándulas accesorias es la secreción de proteínas y cofactores, en especial las semenogelinas, liberadas por la vesícula seminal que contribuyen a la viscosidad del semen como una forma de protegerlo hasta que sea depositado en la vagina, donde las calicreinas ayudaran a que la licuefacción se complete y los espermatozoides puedan iniciar su recorrido. ${ }^{1}$

\section{Etiología de la Prostatitis}

Se denomina prostatitis a la inflamación de la próstata. Esa condición es generalmente originada por procesos infecciosos bacterianos, tratados con antimicrobianos de alto espectro, terapia empleada incluso sin obtener evidencia de la infección, ${ }^{4}$ causando trastornos urinarios y eyaculatorios que afectan la calidad de vida del hombre, la disminución de la viabilidad y la movilidad espermática, ${ }^{5}$ por lo que se considera que las enfermedades que afectan la próstata pueden alterar la fertilidad masculina. ${ }^{1}$

\section{Clasificación de la Prostatitis}

En la - Tabla 1, se presenta la clasificación de prostatitis propuesta por el Instituto Nacional de Salud de los Estados Unidos. ${ }^{4,5}$ Aunque para definir cada tipo de síndrome prostático está especificada la necesidad de evidencia diagnóstica, en la práctica clínica de la urología, la prostatitis sigue caracterizándose por agrupar condiciones extremadamente frecuentes, poco comprendidas, mal tratadas y no investigadas. ${ }^{6}$ De las cuatro categorías reportadas, la categoría III o síndrome de dolor pélvico crónico, es la más prevalente, representando casi el 90\% de los casos y con un alto impacto en la salud física, mental y económica del paciente. ${ }^{6}$ Cerca del $10 \%$ de los casos de síndrome de dolor pélvico crónico es ocasionado por infecciones bacterianas crónicas precedidas por infecciones urinarias mal tratadas. ${ }^{7}$

\section{Epidemiología de la Prostatitis}

Es una enfermedad más común de lo que se cree, cerca del $15 \%$ de los hombres menores de 50 años experimentará síntomas de prostatitis durante su vida, ${ }^{8}$ incluso existe reportes con un mayor porcentaje, entre el $35-50 \%,{ }^{9}$ lo que representa al menos un tercio de la población masculina. ${ }^{6}$

Con una incidencia de 1,26 casos por cada 1000 hombres por año, ${ }^{10}$ esa patología es más frecuente entre la segunda y cuarta década de la vida y de acuerdo a la clasificación, entre el síndrome de dolor pélvico crónico y la prostatitis bacteriana crónica representan alrededor del 90\% de los casos identificados en la práctica clínica, ${ }^{11}$ siendo esa última, responsable del 5 al $10 \%$ de los casos totales de prostatitis, donde el 30\% involucra infecciones urinarias recurrentes. En términos prácticos, los problemas prostáticos inflamatorios representan el $8 \%$ de las consultas al urólogo y el $1 \%$ de las visitas al médico general. ${ }^{2}$

La prevalencia en la población colombiana es desconocida, en países como Estados Unidos, se estima que dos millones de pacientes al año consultan por esa causa, excediendo las consultas por hiperplasia prostática benigna o cáncer de próstata. La prevalencia de prostatitis varía entre 1.,8\% al $65 \%$ en los estudios evaluados, los artículos que reportan la prevalecía de esa entidad clínica son diversos y heterogéneos (-Tabla 2), emplean múltiples metodologías diagnósticas 
Tabla 1 Clasificación de los tipos de prostatitis de acuerdo al Instituto Nacional de Salud de los Estados Unidos

\begin{tabular}{|l|l|l|}
\hline Tipo & Clasificación & Características \\
\hline I & Prostatitis bacteriana aguda & Evidencia de infección bacteriana aguda. \\
\hline II & Prostatitis bacteriana crónica & Evidencia de infección bacteriana recurrente. \\
\hline III & Síndrome de dolor pélvico crónico & Infección no demostrable. \\
\hline A. & Inflamatoria & $\begin{array}{l}\text { Presencia de leucocitos en semen, secreción prostática o en el } \\
\text { sedimento urinario del tercer vaso posterior al masaje } \\
\text { prostático en la prueba de los cuatro vasos de Meares-Stamey. }\end{array}$ \\
\hline B. & No inflamatoria & $\begin{array}{l}\text { No se observa presencia de leucocitos en semen, secreción } \\
\text { prostática o en el sedimento urinario del tercer vaso posterior } \\
\text { al masaje prostático en la prueba de los cuatro vasos de Meares-Stamey. }\end{array}$ \\
\hline IV & $\begin{array}{l}\text { Prostatitis inflamatoria } \\
\text { asintomática }\end{array}$ & $\begin{array}{l}\text { Sin sintomatología. El diagnóstico es incidental durante la biopsia } \\
\text { prostática o por presencia de leucocitos en secreciones prostáticas } \\
\text { obtenidas durante la evaluación de otros desórdenes. }\end{array}$ \\
\hline
\end{tabular}

pero en la gran mayoría, se basan en el reporte de síntomas asociados a prostatitis, de acuerdo al National Institutes of Health Chronic Prostatitis Symtom Index (NIH-CPSI), por lo que hasta la fecha se desconoce el real impacto de esa alteración en la población masculina. ${ }^{12}$

\section{Sintomas de la Prostatitis}

Puede presentar una amplia gama de manifestaciones clínicas, que incluye dolor urogenital, síntomas asociados a infecciones del tracto urinario inferior como dolor durante la micción o el almacenamiento urinario, problemas sicológicos y de disfunción sexual. ${ }^{9}$ En general, los pacientes reportan urgencia urinaria, disuria, dolor pélvico, perineal, genital o de la espalda baja., ${ }^{2,6}$ La evaluación de los síntomas está condicionada por la historia de abuso sexual, físico o emocional, individuos más propensos a manifestar niveles más altos de dolor, ${ }^{9}$ que es de inicio generalmente repentino, con duración promedio de 3 a 6 meses. ${ }^{6}$

\section{Microorganismos}

Aunque sólo del 10\% al 20\% de los casos de prostatitis se han asociado a procesos infecciosos, ${ }^{6,11}$ se cree que esa cifra puede ser mayor debido a las dificultades en el diagnóstico. En general, los procesos infecciosos del tracto genitourinario masculino pueden clasificarse en tres: a) asociados a alteraciones en la microbiota; $b$ ) infecciones recurrentes del tracto urinario y $c$ ) infecciones de transmisión sexual.

\section{Microbiota}

La microbiota ha sido denominada el segundo genoma humano. El cuerpo humano contiene 10 veces más células microbianas que humanas y representa del $1 \%$ al $3 \%$ de la masa corporal total. La dependencia en técnicas de cultivo basadas en la identificación de microbios, los cuales no son cultivables con técnicas tradicionales, hacen que el entendimiento de la fisiología y la fisiopatología sea incompleto. ${ }^{13}$

Cada sitio anatómico del tracto genitourinario puede presentar un recubrimiento con microorganismos saprófitos denominados microbiota, siendo la uretra el sitio con mayor cantidad y variedad microbiana. Entre los colonizadores de la uretra se encuentran Corynebacterium spp., Gardnerella vaginalis, Streptococcus agalactiae, Veillonella parvula, Streptococcus del grupo viridans. Mollicutes, Mycoplasma genitalium, Ureaplasma urealyticum y algunos microorganismos asociados a la microbiota cutánea. Entre los factores que afectan la composición de la microbiota uretral se encuentra la madurez sexual del individuo, si está circuncidado, la frecuencia y tipo de relaciones sexuales, el género y el número de compañeros sexuales, la naturaleza del método de control de la natalidad, el tipo de coito (oral, anal, vaginal). ${ }^{14}$

\section{Infecciones del Tracto Urinario}

En los hombres, a diferencia de las mujeres, la uretra forma parte del sistema reproductivo y es hasta 5 veces más larga que la uretra femenina, haciendo que las infecciones ascendentes de la uretra a los riñones sean menos frecuentes y es un sitio predominantemente aeróbico. La uretra masculina tiene una longitud de $20 \mathrm{~cm}$ y se divide en uretra prostática $(3-4 \mathrm{~cm})$, membranosa $(2 \mathrm{~cm})$ y peneana $(15 \mathrm{~cm})$. En el tracto urinario masculino al igual que el femenino, existen múltiples mecanismos de control de microorganismos que incluyen la descamación del epitelio, el flujo urinario, péptidos antimicrobianos derivados del epitelio, presencia de orina y factores anti adhesión como la glicoproteína de TammHorsfall, IgA y moléculas con actividad antimicrobiana como la urea, anticuerpos y péptidos, además de los componentes antimicrobianos del semen. ${ }^{14}$

Del 10\% de los casos de prostatitis bacteriana crónica, las enterobacterias que causan infecciones urogenitales como Escherichia coli son responsables de cerca del $80 \%$ de los casos, ${ }^{13}$ por lo que los bacilos Gram negativos, cobran gran importancia en la etiología de la inflamación prostática. ${ }^{7}$ Además de su alta prevalencia, un estudio de Ruiz y col., ${ }^{7}$ demuestra que los aislados clínicos de pacientes con prostatitis presentan con mayor frecuencia factores de virulencia como la hemolisina, el factor necrotizante tipo y genes de resistencia a antibióticos en comparación con los aislados de cistitis y pielonefritis. La prueba de Meares y Stemey fue descrita porque aproximadamente el $80 \%$ de los casos diagnosticados por esos investigadores, eran originados por varias cepas de E. coli, el restante $20 \%$ 
Tabla 2 Prevalencia de prostatitis en hombres de la población en general

\begin{tabular}{|c|c|c|c|c|}
\hline $\begin{array}{l}\text { Autores / } \\
\text { año }\end{array}$ & Metodología & Población / país & Prevalencia & Conclusión \\
\hline $\begin{array}{l}\text { Mohanty NK } \\
\text { y col., } \\
1996^{33}\end{array}$ & $\begin{array}{l}\text { Cultivos bacterianos de } \\
\text { biopsia prostática y de orina } \\
\text { pre y postoperatorio. }\end{array}$ & $\begin{array}{l}100 \text { pacientes con } \\
\text { hiperplasia benigna de } \\
\text { próstata sometidos a } \\
\text { prostatectomía. India }\end{array}$ & $42 \%$ & $\begin{array}{l}\text { Crecimiento bacteriano en el } \\
42 \% \text { de las biopsias. Infección } \\
\text { del tracto urinario } \\
\text { preexistente no es un } \\
\text { indicador de infección } \\
\text { prostática. }\end{array}$ \\
\hline $\begin{array}{l}\text { Roberts RO y } \\
\text { col., } 1998^{34}\end{array}$ & $\begin{array}{l}\text { Cuestionario auto- } \\
\text { administrado sobre síntomas } \\
\text { de prostatitis e infección } \\
\text { urinaria, además de revisión } \\
\text { de historias clínicas. }\end{array}$ & $\begin{array}{l}2115 \text { hombres de } 40 \text { a } 79 \\
\text { años. Estados Unidos }\end{array}$ & $9 \%$ & $\begin{array}{l}\text { La prevalencia de prostatitis } \\
\text { es alta, similar a la de la } \\
\text { cardiopatía isquémica y la } \\
\text { diabetes. Los hombres con } \\
\text { diagnóstico previo de } \\
\text { prostatitis tienen mayor } \\
\text { probabilidad de nuevos } \\
\text { episodios. }\end{array}$ \\
\hline $\begin{array}{l}\text { Tanner, M. A } \\
\text { y col., } \\
1999^{35}\end{array}$ & $\begin{array}{l}\text { Análisis de secuencias de } \\
\text { rRNA de fluido prostático. }\end{array}$ & $\begin{array}{l}17 \text { hombres con prostatitis } \\
\text { crónica y } 8 \text { hombres sanos. } \\
\text { Estados Unidos }\end{array}$ & $65 \%$ & $\begin{array}{l}\text { El fluido prostático presenta } \\
\text { un amplio espectro de } \\
\text { especies bacterianas incluso } \\
\text { en hombres sanos. }\end{array}$ \\
\hline $\begin{array}{l}\mathrm{Ku} J \mathrm{H} \text { y col., } \\
2001^{36}\end{array}$ & $\mathrm{NIH}-\mathrm{CPSI}$ & $\begin{array}{l}\text { 6,940 hombres mayores de } \\
20 \text { años seleccionados } \\
\text { aleatoriamente. Corea }\end{array}$ & $6 \%$ & $\begin{array}{l}\text { Hombres con síntomas de } \\
\text { prostatitis experimentan un } \\
\text { impacto negativo sobre la } \\
\text { calidad de vida. }\end{array}$ \\
\hline $\begin{array}{l}\text { Nickel JC y } \\
\text { col., } 2001^{37}\end{array}$ & $\mathrm{NIH}-\mathrm{CPSI}$ & $\begin{array}{l}868 \text { hombres de } 20-74 \text { años. } \\
\text { Canadá }\end{array}$ & $9,7 \%$ & $\begin{array}{l}\text { Los síntomas de prostatitis } \\
\text { crónica son comunes tanto } \\
\text { en hombres jóvenes }(11,5 \%) \\
\text { como en aquellos mayores } \\
50 \text { años }(8,5 \%)\end{array}$ \\
\hline $\begin{array}{l}\text { Screponi, E. } \\
\text { et al, } 2001^{38}\end{array}$ & $\begin{array}{l}\text { Prueba de los cuatro vasos de } \\
\text { Meares-Stamey. }\end{array}$ & $\begin{array}{l}46 \text { pacientes con eyaculación } \\
\text { prematura y } 30 \text { hombres } \\
\text { sanos. Italia }\end{array}$ & $47,8 \%$ & $\begin{array}{l}\text { La prevalencia de } \\
\text { eyaculación prematura } \\
\text { en pacientes con } \\
\text { prostatitis es del } 61,5 \% . \\
\text { La infección prostática } \\
\text { o la inflamación son } \\
\text { factores predisponentes } \\
\text { para la eyaculación } \\
\text { prematura. }\end{array}$ \\
\hline $\begin{array}{l}\text { Collins } \mathrm{MM}_{\mathrm{y}} \\
\text { col., } 2002^{39}\end{array}$ & $\begin{array}{l}\text { Diagnósticos urológicos } \\
\text { reportados por los } \\
\text { profesionales de la salud. }\end{array}$ & $\begin{array}{l}31.681 \text { profesionales de la } \\
\text { salud de } 40 \text { a } 75 \text { años. } \\
\text { Estados Unidos }\end{array}$ & $16 \%$ & $\begin{array}{l}\text { Hombres con antecedentes } \\
\text { de prostatitis tienen } 7,7 \\
\text { veces más probabilidad de } \\
\text { sufrir de hiperplasia benigna } \\
\text { prostática. }\end{array}$ \\
\hline $\begin{array}{l}\text { Roberts RO y } \\
\text { col., } 2002^{40}\end{array}$ & $\mathrm{NIH}-\mathrm{CPSI}$ & $\begin{array}{l}\text { 1,541 hombres } 40 \text { a } 79 \text { años. } \\
\text { Estados Unidos }\end{array}$ & $12 \%$ & $\begin{array}{l}\text { En hombres mayores el NIH- } \\
\text { CPSI puede estar asociado } \\
\text { con hiperplasia prostática } \\
\text { benigna. }\end{array}$ \\
\hline $\begin{array}{l}\text { Tan JK, y col., } \\
2002^{41}\end{array}$ & Encuesta & $\begin{array}{l}1087 \text { hombres de } 21 \text { a } 70 \\
\text { años. Singapur }\end{array}$ & $2,67 \%$ & $\begin{array}{l}\text { La prevalencia de prostatitis } \\
\text { es común y sus síntomas } \\
\text { afectan la calidad de vida. }\end{array}$ \\
\hline $\begin{array}{l}\text { Carver BS, y } \\
\text { col., } 2003^{42}\end{array}$ & $\begin{array}{l}\text { Prevalencia y relación con la } \\
\text { concentración del antígeno } \\
\text { prostático (PSA). }\end{array}$ & $\begin{array}{l}227 \text { hombres seleccionados } \\
\text { aleatoriamente del programa } \\
\text { de detección de cáncer de } \\
\text { próstata. Estados Unidos }\end{array}$ & $32,2 \%$ & $\begin{array}{l}\text { La prevalencia de prostatitis } \\
\text { inflamatoria asintomática } \\
\text { fue del } 32,2 \% \text {. Hombres } \\
\text { con prostatitis asintomática } \\
\text { tienen niveles mayores } \\
\text { de PSA que hombres } \\
\text { sanos. }\end{array}$ \\
\hline $\begin{array}{l}\text { Rizzo M y } \\
\text { col., } 2003^{43}\end{array}$ & $\begin{array}{l}\text { Reportes de urólogos sobre } \\
\text { pacientes atendidos y } \\
\text { encuesta sobre la severidad } \\
\text { de los síntomas. }\end{array}$ & $\begin{array}{l}8503 \text { pacientes atendidos } \\
\text { por } 70 \text { urólogos. Italia }\end{array}$ & $12,8 \%$ & $\begin{array}{l}\text { Prostatitis es un diagnóstico } \\
\text { común entre los urólogos } \\
\text { italianos. }\end{array}$ \\
\hline
\end{tabular}


Tabla 2 (Continued)

\begin{tabular}{|c|c|c|c|c|}
\hline $\begin{array}{l}\text { Autores / } \\
\text { año }\end{array}$ & Metodología & Población / país & Prevalencia & Conclusión \\
\hline $\begin{array}{l}\text { Liang CZ, y } \\
\text { col., } 2004^{44}\end{array}$ & $\begin{array}{l}\text { NIH-CPSI y análisis de las } \\
\text { secreciones prostáticas. }\end{array}$ & $\begin{array}{l}2000 \text { hombres } \\
\text { diagnosticados con } \\
\text { prostatitis crónica. China }\end{array}$ & $\begin{array}{l}49 \% \\
\text { disfunción } \\
\text { sexual }\end{array}$ & $\begin{array}{l}\text { Prostatitis crónica afecta la } \\
\text { calidad de vida. La } \\
\text { prevalencia de disfunción } \\
\text { sexual en pacientes con } \\
\text { prostatitis es alta y está } \\
\text { asociada negativamente con } \\
\text { la edad y la duración de la } \\
\text { enfermedad. }\end{array}$ \\
\hline $\begin{array}{l}\text { Daniels NA y } \\
\text { col., } 2005^{45}\end{array}$ & $\begin{array}{l}\text { Encuesta sobre síntomas de } \\
\text { prostatitis. }\end{array}$ & $\begin{array}{l}5821 \text { hombres mayores de } \\
65 \text { años. Estados Unidos }\end{array}$ & $25 \%$ & $\begin{array}{l}\text { Prostatitis es un factor de } \\
\text { riesgo para el desarrollo de la } \\
\text { hiperplasia prostática } \\
\text { benigna y del cáncer de } \\
\text { próstata. }\end{array}$ \\
\hline $\begin{array}{l}\text { Nickel JC y } \\
\text { col., } 2005^{46}\end{array}$ & $\mathrm{NIH}-\mathrm{CPSI}$ & $\begin{array}{l}3700 \text { hombres activos } \\
\text { sexualmente con síntomas } \\
\text { del tracto urinario bajo } \\
\text { sugestivo o de hiperplasia } \\
\text { prostática benigna en } \\
\text { Europa, Asia, América Latina } \\
\text { y Canadá. }\end{array}$ & $20 \%$ & $\begin{array}{l}\text { Síntomas de prostatitis o de } \\
\text { dolor al eyacular difieren de } \\
\text { los que sólo presentan } \\
\text { infección del tracto urinario } \\
\text { bajo. }\end{array}$ \\
\hline $\begin{array}{l}\text { Nickel JC, y } \\
\text { col., } 2005^{47}\end{array}$ & $\begin{array}{l}\text { Diagnósticos realizados por } \\
\text { los urólogos de la Asociación } \\
\text { Urológica de Canadá y } \\
\text { Quebec. }\end{array}$ & 65 urólogos. Canadá & $2,7 \%$ & $\begin{array}{l}\text { Prostatitis podría no ser tan } \\
\text { frecuente como se ha } \\
\text { considerado. }\end{array}$ \\
\hline $\begin{array}{l}\text { Clemens JQ y } \\
\text { col., } 2006^{48}\end{array}$ & NIH-CPSI & $\begin{array}{l}\text { 1,550 hombres de } 25 \text { a } 80 \\
\text { años. Estados Unidos }\end{array}$ & $11,2 \%$ & $\begin{array}{l}\text { Uno de cada } 9 \text { hombres } \\
\text { presenta síntomas similares a } \\
\text { la prostatitis. }\end{array}$ \\
\hline $\begin{array}{l}\text { Kunishima Y } \\
\text { y col., } \\
2006^{49}\end{array}$ & $\mathrm{NIH}-\mathrm{CPSI}$ & $\begin{array}{l}512 \text { hombres de } 20 \text { a } 79 \\
\text { años. Japón }\end{array}$ & $4,9 \%$ & $\begin{array}{l}\text { La prevalencia de los } \\
\text { síntomas de prostatitis es } \\
\text { similar en hombres menores } \\
\text { como en los mayores de } 50 \\
\text { años. }\end{array}$ \\
\hline $\begin{array}{l}\text { Bartoletti R y } \\
\text { col., } 2007^{50}\end{array}$ & $\begin{array}{l}\text { Prueba de los cuatro vasos de } \\
\text { Meares-Stamey. }\end{array}$ & $\begin{array}{l}5.540 \text { pacientes de } 25 \text { a } 50 \\
\text { años con síntomas de } \\
\text { prostatitis o dolor pélvico } \\
\text { crónico y } 152 \text { hombres } \\
\text { provenientes de parejas } \\
\text { infértiles. Italia }\end{array}$ & $13,8 \%$ & $\begin{array}{l}\text { La prevalencia de prostatitis } \\
\text { crónica o dolor pélvico } \\
\text { crónico es alta y está } \\
\text { relacionada con la dieta, el } \\
\text { consumo de cigarrillo, el } \\
\text { estilo de vida y las } \\
\text { enfermedades } \\
\text { gastrointestinales. }\end{array}$ \\
\hline $\begin{array}{l}\text { Clemens JQ y } \\
\text { col., } 2007^{51}\end{array}$ & $\begin{array}{l}\text { Revisión de las historias } \\
\text { clínicas. }\end{array}$ & $\begin{array}{l}\text { 120,553 hombres. Estados } \\
\text { Unidos }\end{array}$ & $4,5 \%$ & $\begin{array}{l}\text { Prostatitis afecta a } 1 \text { de cada } \\
22 \text { hombres. }\end{array}$ \\
\hline $\begin{array}{l}\text { Trinchieri A y } \\
\text { col., } 2007^{52}\end{array}$ & $\begin{array}{l}\text { Prueba de los cuatro vasos de } \\
\text { Meares-Stamey. }\end{array}$ & $\begin{array}{l}399 \text { pacientes con síntomas } \\
\text { sugestivos de prostatitis. } \\
\text { Italia }\end{array}$ & $34 \%$ a $55 \%$ & $\begin{array}{l}\text { La prevalencia fue de } 34 \% \text { en } \\
\text { hombres con disfunción } \\
\text { eréctil y } 55 \% \text { con disfunción } \\
\text { eyaculatoria. La presencia de } \\
\text { disfunción eréctil o } \\
\text { eyaculatoria es más } \\
\text { frecuente en hombres con } \\
\text { síntomas de prostatitis } \\
\text { severa e inflamatoria. }\end{array}$ \\
\hline $\begin{array}{l}\text { Ejike CE y } \\
\text { col., } 2008^{53}\end{array}$ & $\mathrm{NIH}-\mathrm{CPSI}$ & $\begin{array}{l}1507 \text { hombres de } 20 \text { a } 70 \\
\text { años. Nigeria }\end{array}$ & $12,2 \%$ & $\begin{array}{l}\text { Síntomas de prostatitis } \\
\text { crónica son tan comunes en } \\
\text { Nigeria como en los países en } \\
\text { vías de desarrollo. }\end{array}$ \\
\hline $\begin{array}{l}\text { Korrovits P y } \\
\text { col., } 2008^{54}\end{array}$ & $\begin{array}{l}\text { Espermograma y antígeno } \\
\text { prostático e interleuquina } 6 \\
\text { en plasma seminal. }\end{array}$ & $\begin{array}{l}558 \text { hombres de } 17 \text { a } 19 \\
\text { años. Estonia }\end{array}$ & $6,0 \%$ & $\begin{array}{l}\text { Prostatitis inflamatoria } \\
\text { asintomática tiene una alta }\end{array}$ \\
\hline
\end{tabular}


Tabla 2 (Continued)

\begin{tabular}{|c|c|c|c|c|}
\hline Autores / & Metodología & Población / país & Prevalencia & Conclusión \\
\hline & & & & $\begin{array}{l}\text { prevalencia entre hombres } \\
\text { jóvenes. }\end{array}$ \\
\hline $\begin{array}{l}\text { Liang CZ y } \\
\text { col., } 2009^{55}\end{array}$ & $\mathrm{NIH}-\mathrm{CPSI}$ & $\begin{array}{l}12743 \text { hombres de } 15 \text { a } 60 \\
\text { años. China }\end{array}$ & $8,4 \%$ & $\begin{array}{l}\text { Síntomas de prostatitis son } \\
\text { un problema multifactorial } \\
\text { que afecta a hombres de } \\
\text { todas las edades, relacionada } \\
\text { con el consumo de alcohol, } \\
\text { fumar y el incremento en el } \\
\text { número de relaciones } \\
\text { sexuales. }\end{array}$ \\
\hline $\begin{array}{l}\text { Tripp DA y } \\
\text { col., } 2009^{56}\end{array}$ & $\mathrm{NIH}-\mathrm{CPSI}$ & $\begin{array}{l}264 \text { hombres de } 16 \text { a } 19 \\
\text { años. Canadá }\end{array}$ & $8,3 \%$ & $\begin{array}{l}\text { Es necesario aumentar la } \\
\text { vigilancia y el diagnóstico de } \\
\text { potenciales casos de } \\
\text { prostatitis crónica desde la } \\
\text { adolescencia. }\end{array}$ \\
\hline $\begin{array}{l}\text { Ferris JA y } \\
\text { col., } 2010^{57}\end{array}$ & $\mathrm{NIH}-\mathrm{CPSI}$ & $\begin{array}{l}1346 \text { hombres de } 16 \text { a } 64 \\
\text { años. Australia }\end{array}$ & $7,6 \%$ & $\begin{array}{l}\text { Al menos un tercio de los } \\
\text { australianos experimentan } \\
\text { síntomas de prostatitis } \\
\text { crónica, lo que influye en su } \\
\text { calidad de vida. }\end{array}$ \\
\hline $\begin{array}{l}\text { Liang CZ y } \\
\text { col., } 2010^{58}\end{array}$ & NIH-CPSI y IIEF5 & $\begin{array}{l}12743 \text { hombres de } 15 \text { a } 60 \\
\text { años. China }\end{array}$ & $8,4 \%$ & $\begin{array}{l}\text { Observaron una alta } \\
\text { prevalencia de eyaculación } \\
\text { prematura (15,3\%) en los } \\
\text { pacientes con síntomas de } \\
\text { prostatitis crónica. }\end{array}$ \\
\hline $\begin{array}{l}\text { Hao ZY y } \\
\text { col., } 2011^{59}\end{array}$ & NIH-CPSI y IIEF5 & $\begin{array}{l}12743 \text { hombres de } 15 \text { a } 60 \\
\text { años. China }\end{array}$ & $8,4 \%$ & $\begin{array}{l}\text { Observan una alta } \\
\text { prevalencia de disfunción } \\
\text { eréctil }(40,5 \%) \text { en pacientes } \\
\text { con prostatitis crónica que } \\
\text { aumenta con la edad. }\end{array}$ \\
\hline $\begin{array}{l}\text { Suskind AM y } \\
\text { col., } 2013^{60}\end{array}$ & $\begin{array}{l}\text { Encuesta telefónica sobre } \\
\text { síntomas de cistitis } \\
\text { intersticial, dolor de vejiga o } \\
\text { síndrome de dolor pélvico } \\
\text { crónico o prostatitis. }\end{array}$ & $\begin{array}{l}6072 \text { hombres de } 18 \text { a } 75 \\
\text { años. Estados Unidos }\end{array}$ & $1,8 \%$ & $\begin{array}{l}\text { Puede existir un } \\
\text { subdiagnóstico de los casos } \\
\text { de prostatitis crónica/dolor } \\
\text { pélvico crónico. }\end{array}$ \\
\hline $\begin{array}{l}\text { Wu C y col., } \\
2013^{61}\end{array}$ & $\begin{array}{l}\text { NIH-CPSI y recuento de } \\
\text { leucocitos en líquido } \\
\text { prostático. }\end{array}$ & $\begin{array}{l}1868 \text { hombres de } 19 \text { a } 78 \\
\text { años. China }\end{array}$ & $21,1 \%$ & $\begin{array}{l}\text { La prostatitis inflamatoria } \\
\text { asintomática es altamente } \\
\text { prevalente en China y está } \\
\text { asociada a factores como la } \\
\text { edad, fumar, beber y bajos } \\
\text { niveles de educación. }\end{array}$ \\
\hline $\begin{array}{l}\text { Tang D y col., } \\
2014^{62}\end{array}$ & $\mathrm{NIH}-\mathrm{CPSI}$ & $\begin{array}{l}438 \text { hombres con } \\
\text { eyaculación prematura y } 493 \\
\text { hombres sanos de } 17 \text { a } 65 \\
\text { años. China }\end{array}$ & $32,0 \%$ & $\begin{array}{l}\text { Se observa una alta } \\
\text { prevalencia }(15,8 \%) \text { de } \\
\text { hombres con síntomas } \\
\text { sugerentes de prostatitis } \\
\text { síntomas en pacientes con } \\
\text { eyaculación prematura. }\end{array}$ \\
\hline $\begin{array}{l}\text { Zhang Z, y } \\
\text { col., } 2015^{63}\end{array}$ & NIH-CPSI y IIEF5 & $\begin{array}{l}2790 \text { hombres de } 20 \text { a } 80 \\
\text { años. China }\end{array}$ & $12,4 \%$ & $\begin{array}{l}\text { Se observa una alta } \\
\text { prevalencia de disfunción } \\
\text { eréctil }(61,5 \%) \text { entre los } \\
\text { hombres con síntomas de } \\
\text { prostatitis. }\end{array}$ \\
\hline $\begin{array}{l}\text { Li HJ y col., } \\
2016^{64}\end{array}$ & Meta-análisis. & $\begin{array}{l}11,189 \text { individuos con } \\
\text { prostatitis incluidos en } 24 \\
\text { estudios. }\end{array}$ & & $\begin{array}{l}\text { Se observa una prevalencia } \\
\text { del } 62 \% \text { de disfunción sexual } \\
\text { en pacientes con prostatitis } \\
\text { crónica o dolor pélvico } \\
\text { crónico. }\end{array}$ \\
\hline
\end{tabular}

Abreviaturas: IIEF5, Índice Internacional de Función Eréctil puntuación de 5 (International Index of Erectile Function 5 score); NIH-CPSI, Índice de Síntomas de Prostatitis Crónica - Instituto Nacional de Salud (National Institutes of Health Chronic Prostatitis Symtom Index). 
originado por otras enterobacterias como Klebsielia, Aerobacter, Proteus mivabilis y Pseudomonas, con aproximadamente $15 \%$ de infecciones mixtas. ${ }^{15}$

\section{Infecciones de Transmisión Sexual}

La Chlamydia trachomatis después de las enterobacterias, es el principal agente etiológico responsable de los casos de prostatitis crónica, con alta prevalencia en países en vía de desarrollo como Colombia, donde existe un subregistro debido a la falta de tamizaje de la población en general. ${ }^{16}$ En el Grupo Reproducción se evaluó la presencia de ADN de ese microorganismo en el semen de hombres asintomáticos para infecciones urogenitales sin detectar ningún caso positivo, ${ }^{17}$ sin embargo, queda por evaluar su presencia en hombres con dolor pélvico crónico o síntomas de prostatitis. Adicionalmente, en nuestros ensayos, no observamos efectos sobre la calidad espermática al incubar la bacteria con los espermatozoides en ensayos in vitro sin embargo, $C$. trachomatis puede ser transportado por los espermatozoides humanos y murinos. ${ }^{18}$ El tercer agente etiológico de mayor importancia en la prostatitis bacteriana es Neisseria gonorrhoeae. Su incubación in vitro con los espermatozoides humanos causa disminución de la viabilidad espermática y puede unirse a la cabeza, pieza intermedia y cola del espermatozoide. $^{19}$

Adicionalmente, se ha descrito que las secreciones prostáticas contienen altas concentraciones de zinc que pueden inhibir el crecimiento de múltiples microorganismos como E. coli, C. trachomatis, Candida albicans y Trichomonas spp., a las concentraciones encontradas in vivo. Otro componente antimicrobiano es la espermina y la espermidina, poliaminas con actividad contra amplio rango de microorganismos semenogelina I tiene actividad antibacterial. ${ }^{14}$

\section{Microorganismos Poco Comunes}

Aunque algunos autores los reportan como microbiota urogenital, evaluaciones clínicas revelan una relación estrecha entre las infecciones por Micoplasma hominis y Ureaplasma urealyticum con la infertilidad masculina en infecciones prostáticas afectando la calidad espermática. ${ }^{20}$ En pacientes con tuberculosis renal se han descrito casos de prostatitis por Mycobacterium tuberculosis. ${ }^{15}$ También hay reportes de microorganismos que pueden colonizar el tejido prostático en pacientes inmunosuprimidos como C. albicans, Coccidioides immitis, Blastomyces dermatitidis e Histoplasma capsulatum. $^{9}$

\section{Diagnóstico de la Prostatitis}

El diagnóstico de esa entidad clínica es complejo y aunque se han probado diferentes técnicas, la prueba de los cuatro vasos propuesta por Meares y Stamey en 1972, en la cual se toman muestras de orina y de secreción prostática para ser evaluada por cultivo y por microscopía ${ }^{15}$ sigue siendo considerada como "prueba de oro", no obstante en la práctica médica es poco empleada por el riesgo de bacteriemia, lo engorroso del procedimiento $^{4}$ y porque solo logra demostrar el origen infeccioso en el $10 \%$ de los hombres con síntomas de prostatitis $^{7}$ por lo que nuevos métodos diagnósticos son necesarios, especialmente para los casos asintomáticos. La prueba de los cuatro vasos incluye una primera muestra de orina que representa la uretra, la segunda muestra de orina representa la vejiga, la tercera muestra, que es secreción prostática y el cuarto vaso de orina de la siguiente micción representan la próstata. ${ }^{9}$ Otras modificaciones a esa prueba como la de los dos vasos, en la que se toma una muestra de orina de la porción media de la primera y segunda micción, el hisopado uretral o la modificación Zegarra Montes y col., ${ }^{13}$ que incluye el cambio del tercer vaso de fluido prostático postmasaje al reemplazarlo por semen de eyaculado sin masaje prostático, muestran similar sensibilidad que la prueba de los cuatro vasos, aunque se ha descrito que el cultivo de la secreción prostática revela frecuentemente falsos positivos resultados de la contaminación microbiana o falsos negativos por errores en el muestreo. ${ }^{21}$ El cultivo de semen y de orina individuales revela propiedades diagnósticas limitadas y resultados negativos no excluyen la presencia de prostatitis. $^{13}$

Una alternativa diagnóstica empleada es el espermocultivo, pero no discrimina el sitio de la infección y tanto la prueba de los cuatro vasos como el espermocultivo son inútiles en la detección de bacterias anaerobias e intracelulares. ${ }^{2,7}$ Los agentes etiológicos ligados a la prostatitis son tan diversos que los métodos de cultivo tradicionales no abarcan su diagnóstico en un solo procedimiento $^{22}$ y aunque algunos reportes siguen identificando a las enterobacterias como los principales patógenos responsables de prostatitis, las características fisiológicas de las enterobacterias y su crecimiento pueden opacar la detección del agente infeccioso responsable del cuadro prostático, ${ }^{2}$ por lo que la implementación de técnicas moleculares puede mejorar el diagnóstico.

En nuestra experiencia empleando la técnica del espermocultivo, se observó el crecimiento de una gran diversidad de microorganismos aerobios en altas concentraciones en hombres asintomáticos para infecciones urogenitales, independientemente de la capacidad fértil del individuo. ${ }^{23}$ Empleando técnicas moleculares para la detección de ADN de las principales bacterias responsables de infecciones de transmisión sexual de difícil detección por métodos de cultivo tradicionales, se observó una prevalencia del 3,6\% y del 7,1\%, en la Neisseria gonorrhoeae y Ureaplasma urealyticum, respectivamente, en el semen de 84 individuos asintomáticos para infecciones urogenitales. ${ }^{17}$ Sin embargo, en ambos estudios, no se encontró asociación entre la calidad seminal y la presencia de bacterias en el eyaculado.

Otro método diagnóstico evaluado es la expresión de CD64 en los leucocitos del fluido prostático empleando citometría de flujo. CD64 es una glicoproteína trans membrana de 72-kDa con alta afinidad por el receptor de inmunoglobulinas y un miembro de la superfamilia IgG. ${ }^{24}$ Incluso se ha considerado que la presencia de más de 10 leucocitos por campo de alto poder (40X) en la secreción prostática es indicador de prostatitis. ${ }^{13}$

Cuando es difícil obtener evidencia diagnóstica del cuadro prostático, o como prueba tamiz para la población general, se 
ha empleado el uso de la encuesta NIH-CPSI de síntomas de prostatitis propuesta por el Instituto Nacional de Salud de los Estados Unidos que incluye la localización del dolor, los síntomas miccionales y/o durante la relación sexual, el nivel de dolor y el efecto sobre la calidad de vida. ${ }^{6}$

En términos generales, para el diagnóstico de las cuatro entidades clínicas de prostatitis, se debe realizar un examen físico completo acompañado de cultivos de orina, semen y/o la prueba de los cuatro vasos. Actualmente, se recomienda realizar la prueba de los cuatro vasos modificada que incluya el cultivo de semen en reemplazo de la muestra de fluido prostático para evitar el riesgo de bacteriemia posterior al masaje prostático. Cuando se sospeche de acceso prostático o en los casos en que el tratamiento antibiótico no sea efectivo se puede incluir como ayudas diagnósticas la tomografía computarizada, la resonancia magnética o la ultrasonografía transrectal. La determinación del antígeno prostático sólo se recomienda para el diagnóstico de la prostatitis inflamatoria asintomática cuando la prueba se realiza acompañada de la historia familiar y de los factores de riesgo para cáncer de próstata para su adecuada interpretación. ${ }^{25}$

\section{Impacto Sobre la Calidad Seminal}

Aproximadamente el $15 \%$ de los casos de infertilidad masculina está relacionada con infecciones del tracto genital masculino. ${ }^{20}$ Aunque su relación es controversial, los procesos infecciosos del tracto reproductivo masculino se han catalogado como una importante causa de infertilidad. El punto de mayor interés en el tema de la infertilidad por causas infecciosas, radica en el real impacto de los microorganismos sobre la calidad seminal, inclusive se ha reportado que el síndrome de dolor pélvico inflamatorio y la prostatitis, están asociados a una respuesta inmune acompañada de reacción inflamatoria que incrementa el estrés oxidativo. ${ }^{5}$ La infección bacteriana de los órganos del aparato reproductivo puede afectar la producción de espermatozoides o causar obstrucción de los túbulos que transportan los espermatozoides. ${ }^{26}$ En ensayos previos in vitro de nuestro grupo observamos que tanto las bacterias como los factores que producen durante su metabolismo alteran la calidad seminal ${ }^{27-29}$ incluso demostramos que los espermatozoides humanos pueden transportar bacterias de importancia clínica. ${ }^{18,29}$ Sin embargo, falta evidencia sobre el efecto de la prostatitis bacteriana sobre la calidad espermática. ${ }^{7}$

Los procesos infecciosos acompañados de inflamación afectan la calidad seminal, especialmente por la secreción de varias proteínas regulatorias producidas por los leucocitos y otras células que controlan la inflamación, entre las que se incluyen interleuquinas (IL)-1, IL-2, IL-6 y factor de necrosis tumoral $\alpha$ (TNF $\alpha){ }^{30}$

En pacientes con prostatitis crónica, se ha observado una mayor producción de especies reactivas del oxígeno y menor capacidad antioxidante, lo que induce al daño en la estructura de la cromatina y en la membrana plasmática. ${ }^{5}$

Aunque sea controversial el efecto la prostatitis sobre la fertilidad pero debemos recordar que de los millones de espermatozoides inseminados en el coito en humanos, sólo unos pocos miles llegan a las trompas de Falopio y normalmente sólo un único espermatozoide fertiliza un oocito, ${ }^{31}$ por lo que mejorar la calidad y cantidad de esos competidores eliminando procesos infecciosos e inflamatorios, puede aumentar la probabilidad de alcanzar el éxito reproductivo.

\section{Tratamiento de la Prostatitis}

El uso de terapia antimicrobiana empírica es frecuente sin evidencia de infección, y muchos clínicos basan sus diagnósticos en la historia médica, en la examinación clínica, en el uroanálisis o en la observación microscópica de la secreción prostática e instauran la terapia antimicrobiana antes de obtener evidencia, ${ }^{15,21}$ lo que puede favorecer la dispersión de microorganismos multiresistentes, aunque muchos pacientes sin confirmación de la infección responden a la intervención con antibióticos, lo que posiblemente refleja un efecto anti-inflamatorio o antineuropático del agente antimicrobiano. ${ }^{9}$

El esquema de tratamiento de los pacientes con dolor pélvico inflamatorio crónico o síntomas de prostatitis difiere en los estudios, en general, en que la mayoría de los pacientes reciben esquemas de antibióticos prolongados que van de 14 a 150 días. Los antibióticos de elección por sus características farmacocinéticas, farmacodinámicas y su amplio espectro antimicrobiano, son las fluoroquinolonas y en segundo lugar, los macrolidos. ${ }^{7}$

Una revisión publicada en $2013^{32}$ asegura que la mejor opción de manejo en prostatitis aguda por enterobacterias son las fluoroquinolonas administradas de 4 a 6 semanas. Los tratamientos alternativos incluyen los trimoxazoles, los betalactamicos y las tetraciclinas. Para los microorganismos intracelulares se emplean los macrolidos como la claritromicina y la azitromicina. Sin embargo, el uso repetido de antibióticos como quinolonas debe interrumpirse si no se observa beneficio sobre el control de la infección o si los cultivos no soportan el origen infeccioso. ${ }^{9}$

Un tratamiento poco convencional es el uso de acupuntura por 10 semanas que demuestra casi el doble de posibilidades de disminuir los síntomas en los casos de prostatitis crónica o dolor pélvico crónico, además se debe recordar que los pacientes deben ser manejados acorde con su patrón de síntomas, no existe un protocolo de manejo único para todos los pacientes. ${ }^{9}$

En una revisión publicada recientemente por F.U. Khan y col., ${ }^{25}$ se propone realizar un manejo clínico del paciente con prostatitis de acuerdo a su sintomatología y no por su clasificación, así se consideran seis dominios a tratar: síntomas urinarios, sicosociales, de dolor específico de órgano, síntomas infecciosos, síntomas neurológicos o sistémicos y sensibilidad. Por ejemplo, si un paciente presenta síntomas urinarios, se recomienda realizar modificaciones en la dieta, incluir medicamentos anticolinérgicos para la retención urinaria y piridio que disminuya el dolor, el ardor, la urgencia y la frecuencia miccional. Si presenta síntomas sicosociales se puede incluir el manejo con antidepresivos y terapia sicológica. Los síntomas infecciosos pueden ser tratados con terapia antimicrobiana y los síntomas neurológicos, 
relacionados con el dolor, la sensibilidad sistémica y específica de órgano pueden ser tratados con alfa bloqueadores, gabapentinoides, amitriptilina, neuromodulación, fisioterapia, relajantes musculares y ejercicio.

\section{Conclusión}

La prostatitis es una patología enigmatica con muchos vacíos en el conocimiento. Estudiar ese problema, puede contribuir a mejorar la calidad de vida de los pacientes y reducir los impactos sobre la fertilidad. Sin embargo, se hace necesario conocer la prevalencia de esa alteración en la población general, debido a que aunque la encuesta del NIH-CPS (Índice de Síntomas de Prostatitis Crónica - Instituto Nacional de Salud), es una herramienta útil para determinar los individuos con síntomas similares de prostatitis, los posibles casos requieren mayor evidencia de infección o inflamación a través de la mejora de herramientas diagnósticas, que posibiliten el adecuando tratamiento de los casos disminuyendo así la propagación de infecciones multiresistentes.

\section{Bibliografía}

1 Verze P, Cai T, Lorenzetti S. The role of the prostate in male fertility, health and disease. Nat Rev Urol 2016;13(07):379-386

2 Videčnik Zorman J, Matičič M, Jeverica S, Smrkolj T. Diagnosis and treatment of bacterial prostatitis. Acta Dermatovenerol Alp Panonica Adriat 2015;24(02):25-29

3 Ko EY, Sabanegh ES. The role of nutraceuticals in male fertility. Urol Clin North Am 2014;41(01):181-193

4 Kumar S, Dave A, Wolf B, Lerma EV. Urinary tract infections. Dis Mon 2015;61(02):45-59

5 Shang Y, Liu C, Cui D, Han G, Yi S. The effect of chronic bacterial prostatitis on semen quality in adult men: a meta-analysis of case-control studies. Sci Rep 2014;4:7233

6 Shoskes DA. Chronic prostatitis/chronic pelvic pain syndrome. Springer Science \& Business Media; 2008

7 Wagenlehner FM, Diemer T, Naber KG, Weidner W. Chronic bacterial prostatitis (NIH type II): diagnosis, therapy and influence on the fertility status. Andrologia 2008;40(02):100-104

8 Krieger JN, Riley DE. Bacteria in the chronic prostatitis-chronic pelvic pain syndrome: molecular approaches to critical research questions. J Urol 2002;167(06):2574-2583

9 Rees J, Abrahams M, Doble A, Cooper A; Prostatitis Expert Reference Group (PERG). Diagnosis and treatment of chronic bacterial prostatitis and chronic prostatitis/chronic pelvic pain syndrome: a consensus guideline. BJU Int 2015;116(04):509-525

10 Zhang R, Sutcliffe S, Giovannucci E, et al. Lifestyle and Risk of Chronic Prostatitis/Chronic Pelvic Pain Syndrome in a Cohort of United States Male Health Professionals. J Urol 2015;194(05): $1295-1300$

11 Fu W, Zhou Z, Liu S, et al. The effect of chronic prostatitis/chronic pelvic pain syndrome (CP/CPPS) on semen parameters in human males: a systematic review and meta-analysis. PLoS One 2014;9 (04):e94991

12 Littrup PJ, Lee F, McLeary RD, Wu D, Lee A, Kumasaka GH. Transrectal US of the seminal vesicles and ejaculatory ducts: clinical correlation. Radiology 1988;168(03):625-628

13 Zegarra Montes LZ, Sanchez Mejia AA, Loza Munarriz CA, Gutierrez EC. Semen and urine culture in the diagnosis of chronic bacterial prostatitis. Int Braz J Urol 2008;34(01):30-37, discussion 38-40

14 Wilson M. Bacteriology of humans: an ecological perspective. John Wiley \& Sons; 2009
15 Meares EM Jr, Stamey TA. The diagnosis and management of bacterial prostatitis. Br J Urol 1972;44(02):175-179

16 Alvis N, Mattar S, García J, Conde E, Díaz A. Infecciones de transmisión sexual en un grupo de alto riesgo de la ciudad de Montería, Colombia. Rev Salud Publica (Bogota) 2007;9(01): 86-96

17 Puerta Suárez J, Cardona Maya W. Prevalencia de Chlamydia trachomatis, Neisseria gonorrhoeae y Ureaplasma urealyticum en muestras de semen: efectos sobre la calidad espermática. Urol Colomb 2016;25(03):219-224

18 Puerta Suarez J, Sanchez L, Salazar FC, et al. Chlamydia trachomatis neither damages spermatozoa nor uses them as vector for the transmission of the infection to the female genital tract. Sci Rep 2017;7(01):1126

19 Puerta Suárez J, Cardona Maya WD. Evaluación in vitro del efecto de Neisseria gonorrhoeae y los factores solubles producto de su metabolismo sobre la calidad espermática. Rev Chil Obstet Ginecol 2016;81(03):211-217

20 Huang C, Long X, Jing S, et al. Ureaplasma urealyticum and Mycoplasma hominis infections and semen quality in 19,098 infertile men in China. World J Urol 2016;34(07):1039-1044

21 Ryu JK, Lee SM, Seong DW, et al. Tc-99m ciprofloxacin imaging in diagnosis of chronic bacterial prostatitis. Asian J Androl 2003;5 (03):179-183

22 Krieger JN, Ross SO, Riley DE. Chronic prostatitis: epidemiology and role of infection. Urology 2002;60(6, Suppl):8-12, discussion 13

23 Puerta Suárez J, Villegas Castaño A, Serna Quintana GJ, y col. Espermocultivo: crecimiento bacteriano del eyaculado y su relación con los parámetros seminales. Rev Chil Obstet Ginecol 2015;80(01):33-40

24 Qian L, Li SB, Zhou Y, Teng SJ, Guo JJ. Determination of CD64 for the Diagnosis of Bacterial Chronic Prostatitis. Am J Reprod Immunol 2015;74(04):309-312

25 Khan FU, Ihsan AU, Khan HU, et al. Comprehensive overview of prostatitis. Biomed Pharmacother 2017;94:1064-1076

26 Bhatt CP, Mishra S, Bhatt A, Lakhey M. Bacterial pathogens in semen culture and their antibiotic susceptibility pattern in vitro. Int J Biomed Res 2015;6(11):909-914

27 Cano-Cháves A, Galarzo-Pardo S, Puerta-Suárez J, Giraldo M, Cadavid Á, Cardona-Maya W. Efecto de las bacterias uropatógenas y de los factores solubles de su metabolismo sobre la calidad espermática: Escherichia coli y Enterococcus faecalis. Clin Invest Ginecol Obstet 2016;44(03):106-112

28 Galarzo Pardo S, Cano Cháves MA, Puerta Suarez J, et al. Efecto de los factores solubles de Staphylococcus aureus, Staphylococcus capitis y Staphylococcus epidermidis sobre la funcionalidad espermática. Rev Chil Obstet Ginecol 2015;80(04):316-323

29 Guerrero-Hurtado L, Puerta-Suárez J, Cardona-Maya W. Papel de los espermatozoides en la transmisión de bacterias uropatógenas: Escherichia coli y Enterococcus faecalis. Clin Invest Ginecol Obstet 2016;45(01):2-6

30 Castiglione R, Salemi M, Vicari LO, Vicari E. Relationship of semen hyperviscosity with IL-6, TNF- $\alpha$, IL-10 and ROS production in seminal plasma of infertile patients with prostatitis and prostatovesiculitis. Andrologia 2014;46(10):1148-1155

31 Suarez SS, Pacey AA. Sperm transport in the female reproductive tract. Hum Reprod Update 2006;12(01):23-37

32 Perletti G, Marras E, Wagenlehner FM, Magri V. Antimicrobial therapy for chronic bacterial prostatitis. Cochrane Database Syst Rev 2013;8(08):CD009071

33 Mohanty NK, Jolly BB. Prevalence of bacterial prostatitis in benign prostatic hyperplasia. Indian J Pathol Microbiol 1996;39(02): 111-114

34 Roberts RO, Lieber MM, Rhodes T, Girman CJ, Bostwick DG, Jacobsen SJ. Prevalence of a physician-assigned diagnosis of prostatitis: the Olmsted County Study of Urinary Symptoms and Health Status Among Men. Urology 1998;51(04):578-584 
35 Tanner MA, Shoskes D, Shahed A, Pace NR. Prevalence of corynebacterial 16S rRNA sequences in patients with bacterial and "nonbacterial" prostatitis. J Clin Microbiol 1999;37(06): 1863-1870

$36 \mathrm{Ku} \mathrm{JH}$, Kim ME, Lee NK, Park YH. The prevalence of chronic prostatitis-like symptoms in young men: a community-based survey. Urol Res 2001;29(02):108-112

37 Nickel JC, Downey J, Hunter D, Clark J. Prevalence of prostatitislike symptoms in a population based study using the National Institutes of Health chronic prostatitis symptom index. J Urol 2001;165(03):842-845

38 Screponi E, Carosa E, Di Stasi SM, Pepe M, Carruba G, Jannini EA. Prevalence of chronic prostatitis in men with premature ejaculation. Urology 2001;58(02):198-202

39 Collins MM, Meigs JB, Barry MJ, Walker Corkery E, Giovannucci E, Kawachi I. Prevalence and correlates of prostatitis in the health professionals follow-up study cohort. J Urol 2002;167(03): 1363-1366

40 Roberts RO, Jacobson DJ, Girman CJ, Rhodes T, Lieber MM, Jacobsen SJ. Prevalence of prostatitis-like symptoms in a community based cohort of older men. J Urol 2002;168(06): 2467-2471

41 Tan JK, Png DJ, Liew LC, Li MK, Wong ML. Prevalence of prostatitislike symptoms in Singapore: a population-based study. Singapore Med J 2002;43(04):189-193

42 Carver BS, Bozeman CB, Williams BJ, Venable DD. The prevalence of men with National Institutes of Health category IV prostatitis and association with serum prostate specific antigen. J Urol 2003; 169(02):589-591

43 Rizzo M, Marchetti F, Travaglini F, Trinchieri A, Nickel JC. Prevalence, diagnosis and treatment of prostatitis in Italy: a prospective urology outpatient practice study. BJU Int 2003;92 (09):955-959

44 Liang CZ, Zhang XJ, Hao ZY, Shi HQ, Wang KX. Prevalence of sexual dysfunction in Chinese men with chronic prostatitis. BJU Int 2004; 93(04):568-570

45 Daniels NA, Ewing SK, Zmuda JM, Wilt TJ, Bauer DC; Osteoporotic Fractures in Men (MrOS) Research Group. Correlates and prevalence of prostatitis in a large community-based cohort of older men. Urology 2005;66(05):964-970

46 Nickel JC, Elhilali M, Vallancien G; ALF-ONE Study Group. Benign prostatic hyperplasia (BPH) and prostatitis: prevalence of painful ejaculation in men with clinical BPH. BJU Int 2005;95(04): 571-574

47 Nickel JC, Teichman JM, Gregoire M, Clark J, Downey J. Prevalence, diagnosis, characterization, and treatment of prostatitis, interstitial cystitis, and epididymitis in outpatient urological practice: the Canadian PIE Study. Urology 2005;66(05):935-940

48 Clemens JQ Meenan RT, O’Keeffe-Rosetti MC, Gao SY, Brown SO, Calhoun EA. Prevalence of prostatitis-like symptoms in a managed care population. J Urol 2006;176(02):593-596, discussion 596

49 Kunishima Y, Mori M, Kitamura H, Satoh H, Tsukamoto T. Prevalence of prostatitis-like symptoms in Japanese men: Population-based study in a town in Hokkaido. Int J Urol 2006; 13(10):1286-1289
50 Bartoletti R, Cai T, Mondaini N, et al; Italian Prostatitis Study Group. Prevalence, incidence estimation, risk factors and characterization of chronic prostatitis/chronic pelvic pain syndrome in urological hospital outpatients in Italy: results of a multicenter case-control observational study. J Urol 2007;178 (06):2411-2415, discussion 2415

51 Clemens JQ Meenan RT, O’Keeffe Rosetti MC, Kimes T, Calhoun EA. Prevalence of and risk factors for prostatitis: population based assessment using physician assigned diagnoses. J Urol 2007;178 (4 Pt 1):1333-1337

52 Trinchieri A, Magri V, Cariani L, et al. Prevalence of sexual dysfunction in men with chronic prostatitis/chronic pelvic pain syndrome. Arch Ital Urol Androl 2007;79(02):67-70

53 Ejike CE, Ezeanyika LU. Prevalence of chronic prostatitis symptoms in a randomly surveyed adult population of urbancommunity-dwelling Nigerian males. Int J Urol 2008;15(04): 340-343

54 Korrovits P, Ausmees K, Mändar R, Punab M. Prevalence of asymptomatic inflammatory (National Institutes of Health Category IV) prostatitis in young men according to semen analysis. Urology 2008;71(06):1010-1015

55 Liang CZ, Li HJ, Wang ZP, et al. The prevalence of prostatitis-like symptoms in China. J Urol 2009;182(02):558-563

56 Tripp DA, Nickel JC, Ross S, Mullins C, Stechyson N. Prevalence, symptom impact and predictors of chronic prostatitis-like symptoms in Canadian males aged 16-19 years. BJU Int 2009; 103(08):1080-1084

57 Ferris JA, Pitts MK, Richters J, Simpson JM, Shelley JM, Smith AM. National prevalence of urogenital pain and prostatitis-like symptoms in Australian men using the National Institutes of Health Chronic Prostatitis Symptoms Index. BJU Int 2010;105 (03):373-379

58 Liang CZ, Hao ZY, Li HJ, et al. Prevalence of premature ejaculation and its correlation with chronic prostatitis in Chinese men. Urology 2010;76(04):962-966

59 Hao ZY, Li HJ, Wang ZP, et al. The prevalence of erectile dysfunction and its relation to chronic prostatitis in Chinese men. J Androl 2011;32(05):496-501

60 Suskind AM, Berry SH, Ewing BA, Elliott MN, Suttorp MJ, Clemens JQ. The prevalence and overlap of interstitial cystitis/bladder pain syndrome and chronic prostatitis/chronic pelvic pain syndrome in men: results of the RAND Interstitial Cystitis Epidemiology male study. J Urol 2013;189(01):141-145

$61 \mathrm{Wu} \mathrm{C}$, Zhang Z, Lu Z, et al. Prevalence of and risk factors for asymptomatic inflammatory (NIH-IV) prostatitis in Chinese men. PLoS One 2013;8(08):e71298

62 Tang D, Zhang X, Hao Z, Zhou J, Liang C. Prevalence of prostatitislike symptoms in outpatients with four premature ejaculation syndromes: a study in 438 men complaining of ejaculating prematurely. Int J Clin Exp Med 2014;7(07):1829-1836

63 Zhang Z, Li Z, Yu Q et al. The prevalence of and risk factors for prostatitis-like symptoms and its relation to erectile dysfunction in Chinese men. Andrology 2015;3(06):1119-1124

$64 \mathrm{Li}$ HJ, Kang DY. Prevalence of sexual dysfunction in men with chronic prostatitis/chronic pelvic pain syndrome: a metaanalysis. World J Urol 2016;34(07):1009-1017 